\title{
A DYNAMIC ROUTE DISCOVERY SCHEME FOR HETEROGENEOUS WIRELESS SENSOR NETWORKS BASED ON POLYCHROMATIC SETS THEORY
}

\author{
Dong Wang ${ }^{1}$, Xinheng Wang ${ }^{1}$ and Hong-Hsu Yen ${ }^{2}$ \\ ${ }^{1}$ School of Engineering and Computing, \\ University of the West of Scotland, Paisley, Scotland, UK \\ dong.wang@uws.ac.uk, xinheng.wang@uws.ac.uk \\ ${ }^{2}$ Department of Information Management, \\ Shih Hsin University, Taipei 116, Taiwan \\ hhyen@cc. shu.edu. tw
}

\begin{abstract}
With the development of new networking paradigms and wireless protocols, nodes with different capabilities are used to form a heterogeneous network. The performance of this kind of networks is seriously deteriorated because of the bottlenecks inside the network. In addition, because of the application requirements, different routing schemes are required toward one particular application. This needs a tool to design protocols to avoid the bottlenecked nodes and adaptable to application requirement. Polychromatic sets theory has the ability to do so. This paper demonstrates the applications of polychromatic sets theory in route discovery and protocols design for heterogeneous networks. From extensive simulations, it shows the nodes with high priority are selected for routing, which greatly increases the performance of the network. This demonstrates that a new type of graph theory could be applied to solve problems of complex networks.
\end{abstract}

\section{KEYWORDS}

Dynamic routing, heterogeneous networks, wireless sensor networks, polychromatic sets

\section{INTRODUCTION}

Wireless communications play an important role in modern communications because of excellent performance and capability in mobility. With the development of wireless protocols and the new paradigm of networking, sensor nodes equipped with different protocols may join together to form a heterogeneous network. Because of the difference in wireless protocols and the ability of communications of these heterogeneous sensor nodes, the design of routing protocols inside the heterogeneous networks is becoming difficult.

Natarajan Meghanathan et al. (Eds) : NETCOM, NCS, WiMoNe, GRAPH-HOC, SPM, CSEIT - 2016 pp. 103-115, 2016. (C) CS \& IT-CSCP 2016

DOI : $10.5121 /$ csit.2016.61510 
Graph theory is a common approach used to design protocols for networking. Traditionally only one metric is considered when a routing protocol is designed. However, in a heterogeneous network, multiple metrics need to be considered. Therefore, it is essential to investigate new types of graph theories to design algorithms and protocols for modern complex heterogeneous networks.

Polychromatic Sets (PS) theory has the ability to consider multiple properties of a set member and describe the relations among the properties, which enables it an appropriate tool to design efficient protocols for networking. Previous research has demonstrated that it could be applied to design protocols for large-scale sensor networks [1]. In this paper, the research work has been extended to heterogeneous networks to investigate the possibility of applying polychromatic sets theory to design protocols for more complex networks.

The performance requirement of a network is always different under various application situations. For example, in the situation of using a wireless sensor network to monitor the fire in a forest, the network should keep its nodes to work as long as possible. Therefore, energy saving is a key task to extend the lifetime of the network. In this case, during normal monitoring process, most devices could be turned into the sleep mode and only a few devices work regularly in order to decrease the energy consumption. In case of detecting the fire, the network is required to operate with high throughput and reliably. Therefore, the routing protocols of such a wireless sensor network need to be adapted to the application, which means the routing protocol should be dynamic.

A heterogeneous sensor network consists of different sensor nodes, such as temperature sensor, humidity sensor, smog sensor and camera sensor. These devices may operate differently in terms of frequency, bandwidth and protocol. Frequency, bandwidth, wireless protocol, energy level of the nodes, and location are inherent characteristics of a sensor node. Throughput, delay, package delivery ratio are performance parameters of the network. All these parameters are defined as the properties of the sensor nodes. Some of them are used as metrics to design the routing protocols in this paper.

In this paper, eight network property parameters, namely location, frequency, residual energy, energy consumption, power mode, bandwidth, throughput, and delay, are considered to design the routing protocols in heterogeneous wireless sensor networks based on PS theory. A dynamic route discovery scheme is proposed for designing the routing protocols. Simulation results demonstrate that performance requirements could be reached under different applications.

The remainder of this paper is organized as follows. Section 2 introduces the related research work. Definitions of metrics used in this paper are described in Section 3. The methodology to define the route discovery based on PS theory is presented in Section 4. PS theory and design of new routing protocols based on PS theory are presented in Section 5. Simulation results are presented in Section 6 with results analysis. Finally this paper is concluded in Section 7.

\section{RELATED WORK}

Traditional graph theory is not suitable for designing protocols for complex modern networks. New types of graph theories, such as random geometric graphs theory [2], directed graphs network model [3] and distributed graph theory [4], were developed to design the protocols. 
However, even these new developed graph theories are not fully functional in terms of designing protocols by considering both the properties of the network nodes and links. Limitations of these graph theories are still obvious.

Polychromatic graph based on PS theory is different from previously developed graphs. PS theory has the ability to describe multiple properties of a set member, which makes it an ideal tool to consider the properties of both the network nodes and links. Previous work in [1] demonstrated the feasibility.

Currently, there is little research conducted on multiple metrics aware routing for wireless networks. Normally, for wireless sensor networks, energy consumption [5] or QoS based routing [6] are two major concerns. Multi-constrained QoS routings [6] is a hot research topic, where quite a lot of research has been done. For example, Ben-Othman et al. proposed an energy and QoS aware multi-path routing for wireless sensor networks [7]; Yao et al. provided an energyefficient, delay-aware and lifetime-balanced data collection [8]; Maurya et al. presented an energy efficient routing for heterogeneous wireless sensor networks by considering distance, energy and load parameters of a network [9]. However, even for multi-constraints QoS routing, the parameters used for designing routing protocols are predefined and, therefore, it is difficult to adapt to the dynamic nature of modern heterogeneous wireless networks.

The aim of the research work presented in this paper is to address this challenge and design a dynamic routing protocols towards application requirements.

\section{DEFINITION OF NETWORK PARAMETERS}

As shown in figure 1, eight main parameters of a heterogeneous wireless sensor network are considered for designing routing protocols, including location, frequency, node residual energy, energy consumption level, power mode, bandwidth, throughput, and delay.

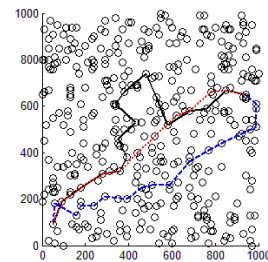

(a) Location

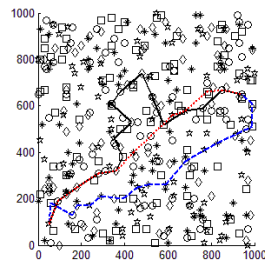

(e) Power Mode

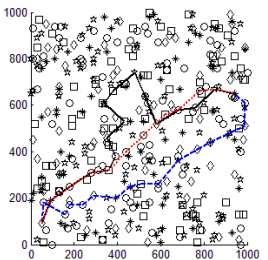

(b) Frequency \& Connectivity *

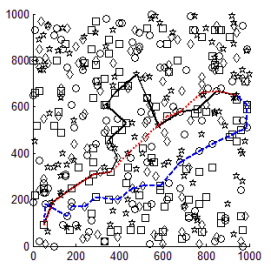

(f) Bandwidth

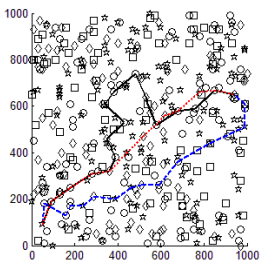

(c) Residual Energy

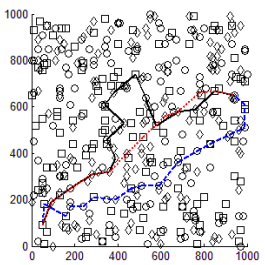

(g) Throughput

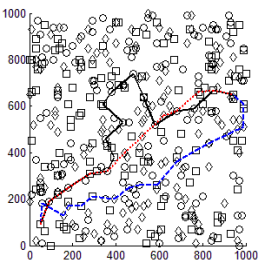

(d) Energy Consumpiton

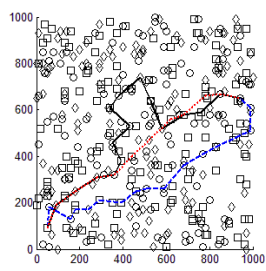

(h) Delay

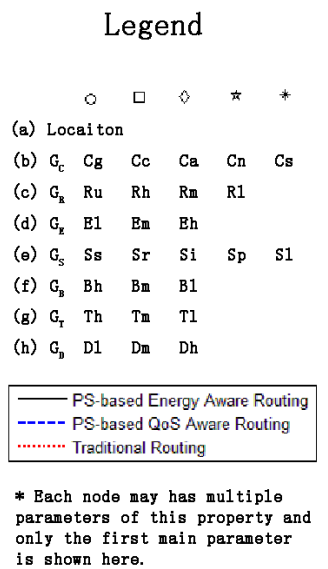

on1y the first
is shown here.

Figure 1. Route Discovery based on Different Metrics 


\section{Location}

The location of a node is a popular metric in wireless sensor networks for designing geographical routing protocols. Based on GPS or other localization techniques [10], it is easy for the nodes to acquire the information of their location. A node's location is defined as $L\left(x_{L}, y_{L}, z_{L}\right)$ in the three-dimensional plane or $L\left(x_{L}, y_{L}\right)$ in the two-dimensional plane.

\section{Frequency}

In a heterogeneous network, network nodes may be equipped with facilities operating at different frequencies. The nodes can only connect with other nodes operating on the same frequency band and protocol. Some of these nodes are able to operate at multiple frequencies. In this scenario, five popular protocols are considered and defined as: $C_{g}, 5 \mathrm{GHz}$ (based on IEEE 802.11ac protocol); $C_{c}, 2.4 \mathrm{GHz}$ (based on IEEE 802.11 n protocol); $C_{a}, 2.4 \mathrm{GHz}$ (based on IEEE $802.11 \mathrm{~g}$ protocol); $C_{n}, 2.4 \mathrm{GHz}$ (based on IEEE $802.11 \mathrm{~b}$ protocol); and $C_{s}, 2.4 \mathrm{GHz}$ (based on IEEE 802.15.4 protocol).

\section{Residual Energy}

Since most of the sensor nodes are powered by battery, energy consumption is one of the biggest concerns for wireless sensor networks. In order to extend the lifetime of the networks, sensor nodes with high residual energy are more often used during data transmission. The low residual energy nodes are protected and less used to save the energy. In order to meet the requirement of applying set theory in designing protocols, four levels of nodes' residual energy are defined as: $R_{u}$, Unlimited Residual Energy; $R_{h}$, High Residual Energy; $R_{m}$, Medium Residual Energy; and $R_{l}$, Low Residual Energy.

\section{Energy Consumption}

The energy consumption of different types of nodes in a heterogeneous network is always different. The energy consumption of nodes is defined with three levels: $E_{l}$, Low Energy Consumption; $E_{m}$, Medium Energy Consumption; and $E_{h}$, High Energy Consumption.

\section{Power Mode}

In wireless sensor networks, network nodes have four power modes: sending, receiving, idle, and sleep. In some special applications, another mode, silence, is also considered. It could save the transfer time by considering and choosing the relay nodes working on high priority modes (like idle) than those nodes working on low priority modes (like sending). The five parameters of power mode are defined as: $S_{s}$, Sending; $S_{r}$, Receiving; $S_{i}$, Idle; $S_{p}$, Sleep; and $S_{l}$, Silence.

\section{Bandwidth}

Bandwidth is an important metric to describe the capability of communications in wireless networks. The bandwidth of links connecting various devices is normally different. In 
heterogeneous networks, the largest residual bandwidth between various devices is defined with four parameters: $B_{e}$, Extra-high Bandwidth; $B_{h}$, High Bandwidth; $B_{m}$, Medium Bandwidth; and $B_{l}$, Low Bandwidth.

\section{Throughput and Delay}

The throughput and delay of networks are two important metrics to describe the QoS of wireless networks. In this scenario, the available throughput is defined with three parameters: $T_{h}$, High Throughput; $T_{m}$, Medium Throughput; and $T_{l}$, Low Throughput. The delay of networks is also defined with three parameters: $D_{l}$, Low Delay; $D_{m}$, Medium Delay; and $D_{h}$, High Delay.

In summary, eight main metrics of heterogeneous wireless sensor networks have been collected and introduced. In next section, the multiple metrics aware heterogeneous network will be defined with this eight metrics by PS theory.

\section{NETWORK DEFINITIONS BASED ON PS}

In this section, the definition of the networks based on PS theory is described.

\subsection{Nodes Set}

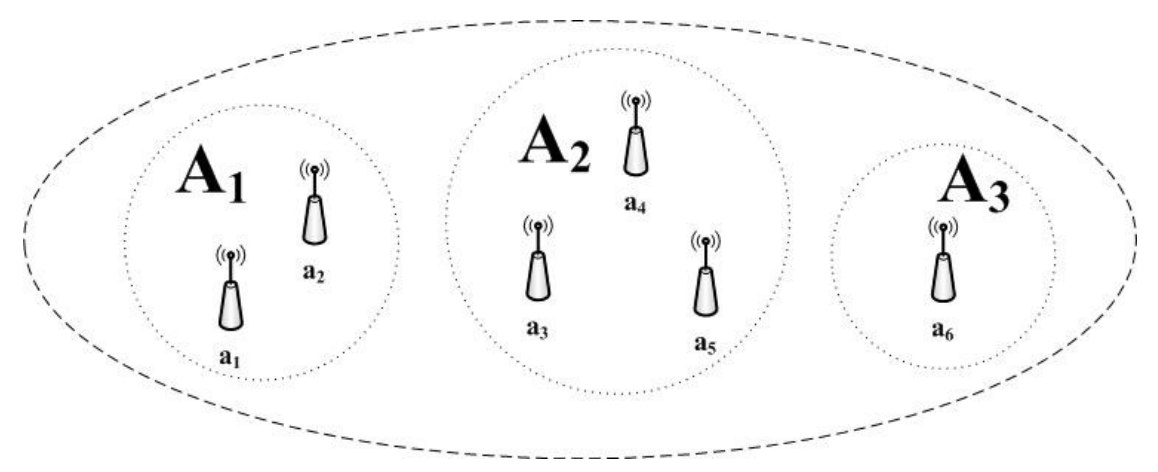

Figure 2. Illustration of a Network and Grouping

As shown in figure 2, a network consists of six nodes. The nodes set $A$ is defined as:

$$
A=\left\{a_{1}, a_{2}, \Lambda, a_{i}, \Lambda, a_{n}\right\}
$$

where $n$ is the number of nodes. These nodes can be divided into several groups based on their locations or other features. For example, in the network shown in figure 2, node $a_{1}$ and $a_{2}$ belong to set $A_{1}$, node $a_{3}, a_{4}$ and $a_{5}$ belong to set $A_{2}$, and node $a_{6}$ belongs to set $A_{3}$.

\subsection{Colour Set}

In PS theory, each property is defined as one individual colour. A colour set $F$ is defined to include all the individual colours. 


$$
F=\left\{f_{1}, f_{2}, \Lambda, f_{j}, \Lambda, f_{n_{F}}\right\}
$$

where $f_{j}$ is an individual colour and $n_{F}$ is the number of colours/properties defined in $F$.

In the case of this paper, all the colours, excluding locations, are presented as:

$$
F=\left\{C_{g}, C_{c}, C_{a}, C_{n}, C_{s}, R_{u}, R_{h}, R_{m}, R_{l}, E_{l}, E_{m}, E_{h}, S_{s}, S_{r}, S_{i}, S_{p}, S_{l}, B_{h}, B_{m}, B_{l}, T_{h}, T_{m}, T_{l}, D_{l}, D_{m}, D_{h}\right\}
$$

These colours could be also classified into several groups, as:

$$
F\left\{\begin{array}{l}
G_{C}=\left\{C_{g}, C_{c}, C_{a}, C_{n}, C_{s}\right\} \\
G_{R}=\left\{R_{u}, R_{h}, R_{m}, R_{l}\right\} \\
G_{E}=\left\{E_{l}, E_{m}, E_{h}\right\} \\
G_{S}=\left\{S_{s}, S_{r}, S_{i}, S_{p}, S_{l}\right\} \\
G_{B}=\left\{B_{h}, B_{m}, B_{l}\right\} \\
G_{T}=\left\{T_{h}, T_{m}, T_{l}\right\} \\
G_{D}=\left\{D_{l}, D_{m}, D_{h}\right\}
\end{array}\right.
$$

In addition, location is utilized for the geographical routing, which will be discussed in next section.

Based on the definition of individual colours, the relations of nodes and their properties/colours can be expressed as a matrix:

$$
\begin{aligned}
& \begin{array}{ccccc}
C_{g} & \mathrm{~K} & f_{j} & \mathrm{~K} & D_{h}
\end{array} \\
& {[A \times F]=\left[\begin{array}{ccccc}
c_{11} & \mathrm{~K} & c_{1 j} & \mathrm{~K} & c_{1,26} \\
\mathrm{~K} & \mathrm{~K} & \mathrm{~K} & \mathrm{~K} & \mathrm{~K} \\
c_{i 1} & \mathrm{~K} & c_{i j} & \mathrm{~K} & c_{i, 26} \\
\mathrm{~K} & \mathrm{~K} & \mathrm{~K} & \mathrm{~K} & \mathrm{~K} \\
c_{n 1} & \mathrm{~K} & c_{n j} & \mathrm{~K} & c_{n, 26}
\end{array}\right] \begin{array}{l}
a_{1} \\
\mathrm{~K} \\
a_{i} \\
\mathrm{~K} \\
a_{n}
\end{array}} \\
& c_{i j}= \begin{cases}1, & f_{j} \in F_{j} \\
0 & \end{cases}
\end{aligned}
$$

$n_{F}=26$ based on the definitions of all the properties.

\subsection{Unity Colour Set}

In a network, some nodes share some common properties. These common properties are defined as a unity colour set $F_{f}$, which is a subset of colour set $F$ as:

$$
\left\{\begin{array}{c}
F_{f}=\left\{f_{f 1}, f_{f 2}, \mathrm{~K}, f_{f j}, \mathrm{~K}, f_{f o}\right\} \\
F_{f} \subset F
\end{array}\right.
$$


The selected nodes are also defined as a set $A_{f}$, which is a subset of nodes set $A$. The relations between sets $F_{f}$ and $A_{f}$ is presented as a matrix, similar to sets $A$ and $F$ :

$$
\begin{gathered}
\left.f_{f 1} \times F_{f}\right]=\left[\begin{array}{lllll}
c_{11} & \mathrm{~K} & c_{1 j} & \mathrm{~K} & c_{1 o} \\
\mathrm{~K} & \mathrm{~K} & \mathrm{~K} & \mathrm{~K} & \mathrm{~K} \\
c_{i 1} & \mathrm{~K} & c_{i j} & \mathrm{~K} & c_{i o} \\
\mathrm{~K} & \mathrm{~K} & \mathrm{~K} & \mathrm{~K} & \mathrm{~K} \\
c_{m 1} & \mathrm{~K} & c_{m j} & \mathrm{~K} & c_{m o}
\end{array}\right] \begin{array}{l}
a_{1} \\
\mathrm{~K} \\
a_{i} \\
\mathrm{~K} \\
a_{m}
\end{array} \\
c_{i j}= \begin{cases}1, f_{f j} \in F_{j} \\
0\end{cases}
\end{gathered}
$$

\subsection{Available Paths Set}

If two nodes share the same properties, a link/path could be established between these two nodes. The total paths available inside a network could be defined as :

$$
\begin{aligned}
& F\left(A_{k 1}\right) \wedge F\left(A_{k j}\right) \wedge F\left(A_{k l}\right) \\
& {[A \times A(F)]=\left[\begin{array}{ccccc}
c_{11} & \Lambda & c_{1 j} & \Lambda & c_{1 l} \\
\Lambda & \Lambda & \Lambda & \Lambda & \Lambda \\
c_{i 1} & \Lambda & c_{i j} & \Lambda & c_{i l} \\
\Lambda & \Lambda & \Lambda & \Lambda & \Lambda \\
c_{m 1} & \Lambda & c_{m j} & \Lambda & c_{m l}
\end{array}\right] \quad \begin{array}{l}
a_{1} \\
a_{i} \\
\Lambda \\
a_{m}
\end{array}} \\
& c_{i j}= \begin{cases}1 & a_{i} \in A_{k j} \\
0 & \end{cases}
\end{aligned}
$$

where $F\left(A_{k j}\right)$ is a set of colours that a group of nodes $A_{k j}$ in the corresponding column have all of those colours, defined as:

$$
\left\{\begin{array}{c}
F\left(A_{k j}\right)=\left\{f_{k 1}, f_{k 2}, \Lambda, f_{k i}, \Lambda, f_{k o}\right\} \\
F\left(A_{k j}\right) \subset F_{f} \\
\forall f_{k i} \subset F\left(A_{k j}\right): \\
{\left[A_{k j} \times f_{k i}\right]=\left[\begin{array}{lllll}
1 & \Lambda & 1 & \Lambda & 1
\end{array}\right]^{T}}
\end{array}\right.
$$

where $\left\lfloor A_{k j} \times f_{k i}\right\rfloor$ is an all-ones matrix.

A route could be established by using the available paths from (9). Algorithm 1 can be used to find the available paths $[A \times A(F)]$. In algorithm $1,[F \times F(A)]$ is the conjunction of different nodes' properties, $\left[F_{m} \times A\left(F_{m}\right)\right]$ is a rank of node's properties, $F_{m}$ and $F_{m n l}\left(a_{i}\right)$ are the tags whether $a_{i}$ has all the properties $F_{m n l}$ or not. Based on this algorithm, 7 parameters are required 
for input: a set of nodes $A_{f}$, a set of colours $F_{f}$, a set of nodes' properties $\left\lfloor A_{f} \times F_{f}\right\rfloor$, number of node groups $n_{A}$, node groups $A_{1}, \Lambda, A_{n_{A}}$, number of property groups $n_{F}$ and metric groups $G_{1}$, $\Lambda, G_{n_{F}}$. The set of selected nodes $\left\lfloor A \times A(F)^{\prime}\right\rfloor$ and the parallel set of their matching metrics $F\left(A_{k}\right)$ ' are output.

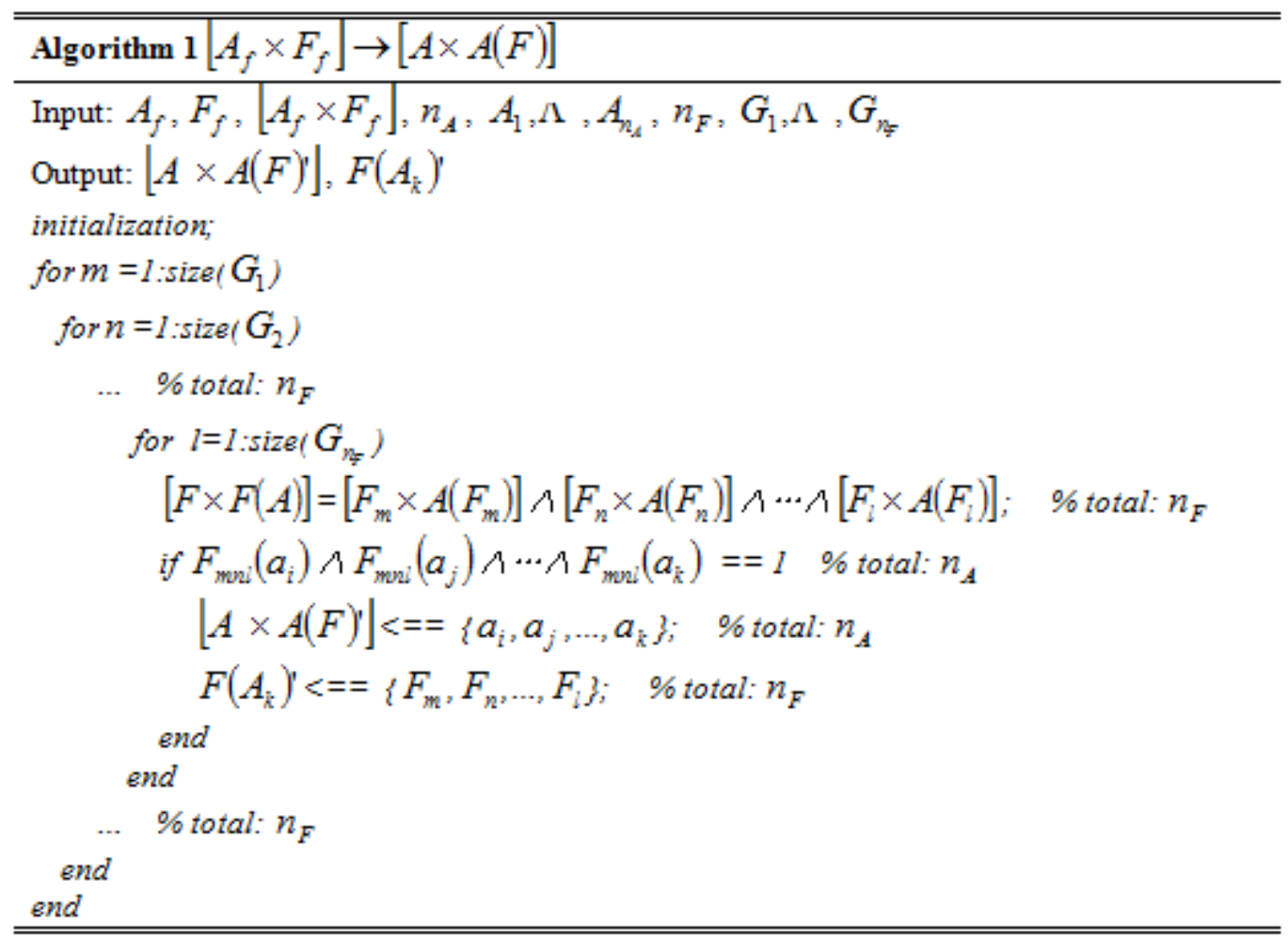

\section{GEOGRAPHICAL ROUTING BASED ON PS}

Location based routing [11] is a popular routing scheme for multi-hop networks including heterogeneous wireless sensor networks. The data packets are transmitted to the destination node(s) based on their location rather than their ID or logical address. In this paper, location based routing is also applied to demonstrate the performance of routing protocols based on PS theory.

\subsection{Greedy Forwarding Routing}

As shown in figure 3, a general location based routing, greedy forwarding routing (GFR) [12], is selected for comparison. In traditional GFR, every node broadcasts its location information to its neighbour nodes so that the current node knows the location information of other nodes in its signal coverage area. The location of the destination node is also known. 


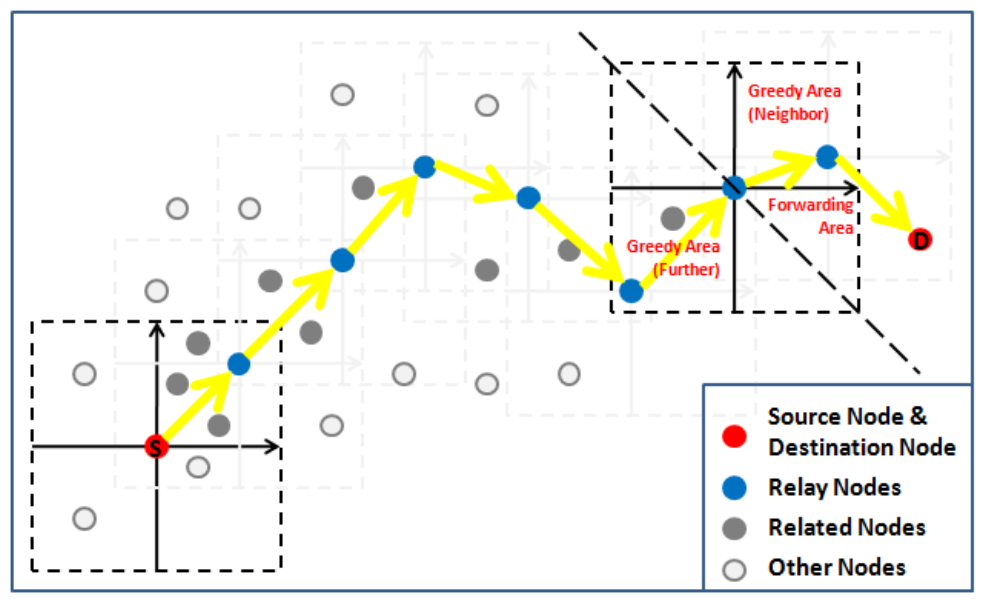

Figure 3. Greedy Forwarding Routing

The area around a node is divided into four zones based on the distance to the destination. As shown in figure 3, the nearest to the destination node is the forwarding area and the other two near areas are neighbour greedy areas and further greedy area, respectively. Whenever a route is to be established, the node in forwarding area which is the nearest to the destination should be selected as the next hop node. If there is no such a suitable node in the forwarding area, the suitable node in neighbour greedy areas should be selected. The node in further greedy area will be selected as a last resolution if necessary.

\subsection{Next Hop Selection Based on PS}

Route discovery based on PS theory is different from traditional. As shown in figure 4, when the destination node is not in the signal coverage area of current node, the current node is classified as a node group $A_{1}$ by itself and the nodes in the considered area are classified as node group $A_{2}$. After collecting the properties of these nodes in $A_{2}$, the nodes having the expected properties are included in an available paths set. The node which is the nearest to the destination node in this set is selected as the next hop node. In figure 4 , node $a_{3}$ with high priority property is selected as the next hop node even though node $a_{2}$ with low priority in the same area is much nearer to the destination node than node $a_{3}$.

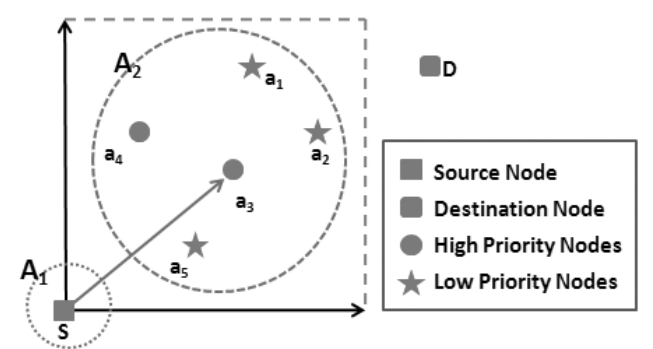

Figure 4. PS-based Metrics Aware Next Hop Node Selection 


\section{Simulations AND ANALYSIS}

A simulation platform based on PS theory is built and developed for simulating the heterogeneous wireless sensor networks in Matlab. 400 1000 nodes are deployed in a $1000 \mathrm{~m} \times 1000 \mathrm{~m}$ area randomly. These nodes have eight different properties as defined in section 3. In a simplified simulation, we assume that all the nodes can connect with the neighbour nodes around it based on traditional GFR. Energy efficient routing is designed by considering three metrics (Residual Energy, Energy Consumption and Power Mode) and QoS aware routing is designed by considering Bandwidth, Throughput and Delay. The discovery radius of each node is set as $100 \sqrt{2} \mathrm{~m}$. The optimized routes are selected based on energy efficient routing, QoS aware routing and traditional GFR routing, which are shown in figure 1 . The performance of the routing protocols is evaluated by the number of participation nodes with different priorities, throughput and delay.

\subsection{Application Ratio of Nodes with Different Metrics}

In a wireless sensor network, nodes are classified into different priority groups based on requirements. For example, nodes with high residual energy and low energy consumption level are classified as high priority nodes and nodes with high throughput are also classified as high priority nodes. Three levels, namely high priority, medium priority and low priority, are used to classify the nodes. The number of nodes at each priority group is similar. Figure 5 shows the results of number of nodes selected for routing with different priorities based on PS routing and traditional GFR routing. In GFR, the ratios of applying nodes with different priorities are similar, between $35 \%$ and $45 \%$. In PS-based routing, the application of nodes with high priority is more than $60 \%$. At the same time, few of low priority nodes are utilized and none of low priority node is applied when the number of nodes is more than 700. The PS-based routing improves the application of high priority nodes and decreases the application of low priority nodes.

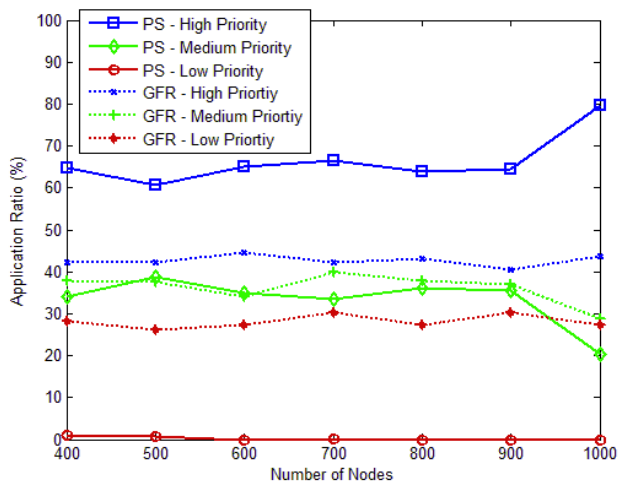

(a) Energy Aware Routing

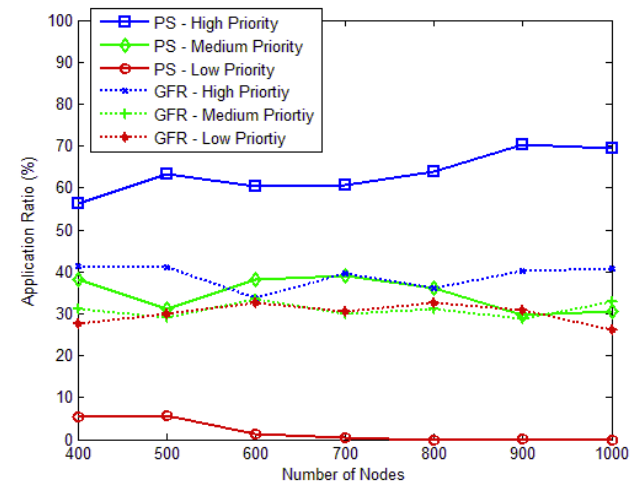

(b) QoS Aware Routing

Figure 5. Application Ratio of Nodes with Different Priority

Figure 6 shows the ratios of numbers of nodes with different priority under other six evaluation metrics. For all the scenarios, PS based routing significantly increased the ratios of applying high priority nodes and decreased the ratios of applying low priority nodes. This is one advantage of PS based routing protocols. 


\subsection{Throughput and Delay}

Figure 7 shows the results of throughputs and figure 8 shows the results of delay for protocols based on GFR and PS. In figure 7, the bandwidth of some nodes in the simulated network is $11 \mathrm{Mbps}$, such as IEEE $802.11 \mathrm{~b}$ protocol, the average throughput of GFR network is limited by these nodes. However, PS-based routing is possible to avoid using these nodes with low bandwidth and then improves the overall network throughput.

The same reason applies to delay. Since PS has the ability to select nodes with short delay, the overall network delay is shorter than GFR routing. The results are shown in figure 8.

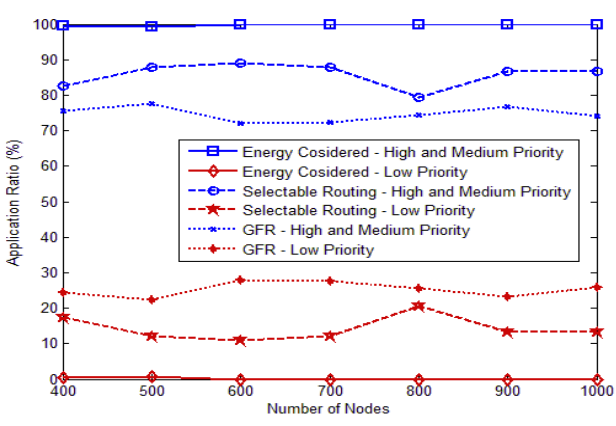

(a) Different Residual Energy Nodes

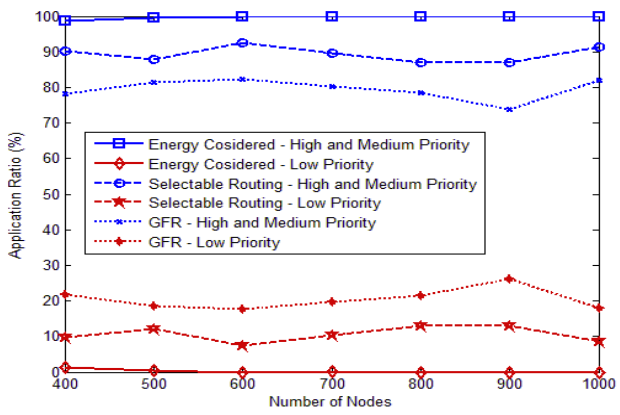

(c) Different Power Mode Modes

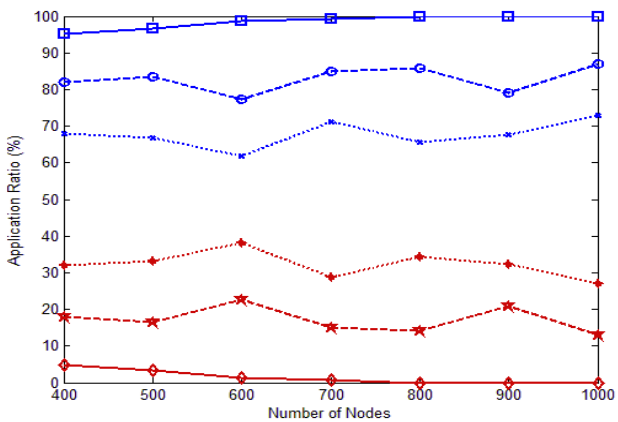

(e) Different Throughput Nodes

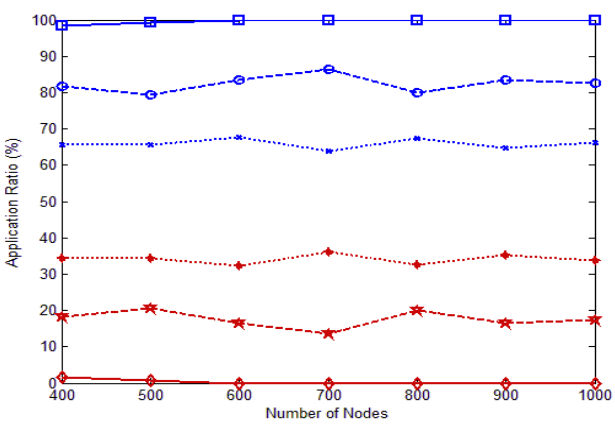

(b) Different Energy Consumption Nodes

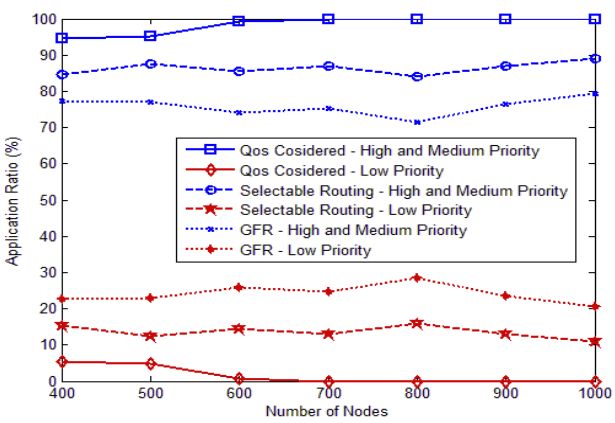

(d) Different Bandwidth Nodes

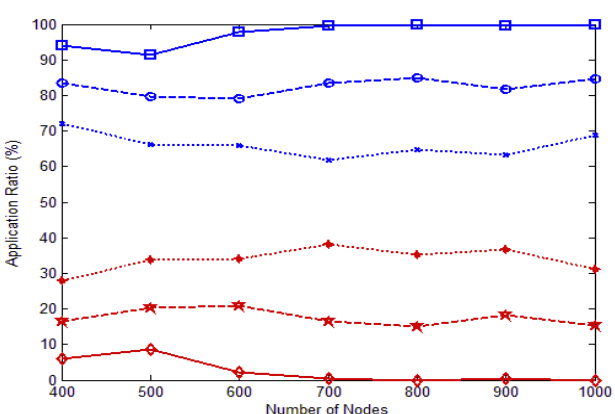

(f) Different Delay Nodes

Figure 6. Application of Nodes with Different Metrics on Different Modes 


\section{CONCLUSION}

In this paper, a PS-based route discovery and routing protocol design are proposed to improve the performance of the network. Multiple metrics of heterogeneous networks are considered in this network model. The performance of heterogeneous networks was improved prominently. This demonstrates the feasibility of applying PS theory in designing protocols for heterogeneous networks.

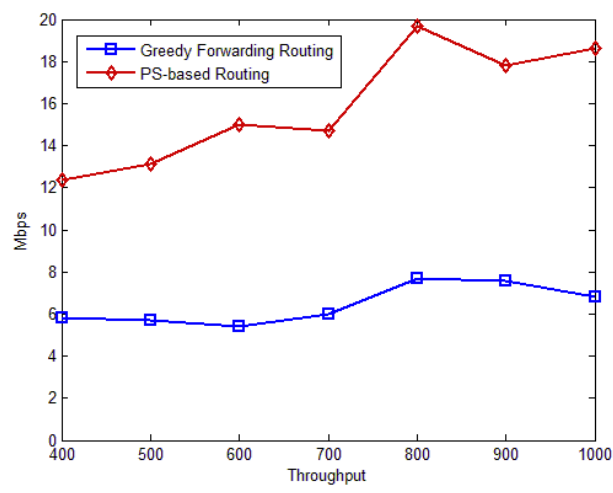

Figure 7. Throughput

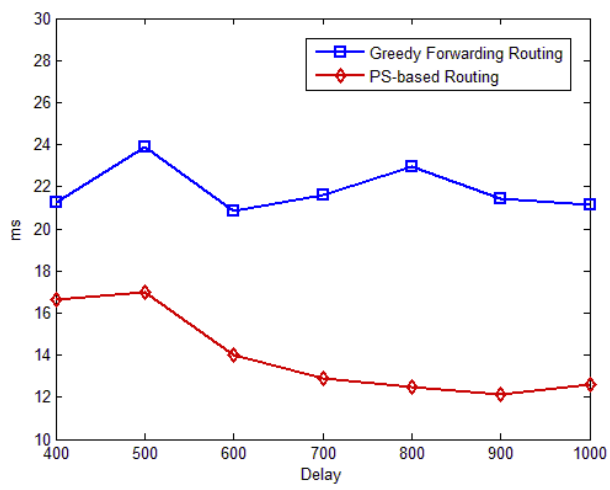

Figure 8. Delay

\section{REFERENCES}

[1] Xinheng Wang \& Shancang Li, (2013) "Scalable routing modeling for wireless ad hoc networks by using polychromatic sets", IEEE Systems Journal, Vol. 7, No. 1, pp50-58.

[2] M. Haenggi, J. G. Andrews, F. Baccelli, O. Dousse\& M. Franceschetti, (2009) "Stochastic geometry and random graphs for the analysis and design of wireless networks", IEEE Journal on Selected Areas in Communications, Vol. 27, No. 7, pp1029-1046.

[3] Yanhua Li \& Zhi-Li Zhang, (2010) "Random walks on digraphs: A theoretical framework for estimating transmission costs in wireless routing", IEEE INFOCOM, pp1-9.

[4] Qing Chen, Qian Zhang \& Zhisheng Niu, (2009) "A graph theory based opportunistic link scheduling for wireless ad hoc networks", IEEE Transactions on Wireless Communications, Vol. 8, No. 10, pp5575-5585.

[5] Xiao Chen, Zanxun Dai \& Hongchi Shi, (2013) "EgyHet: An energy-saving routing protocol for wireless heterogeneous sensor networks", IEEE ICNC, pp778-782.

[6] Xiaoxia Huang \& Yuguang Fang, (2008) "Multiconstrained QoS multipath routing in wireless sensor networks", Wireless Networks, Vol. 14, No. 4, pp465-478.

[7] Jalel Ben-Othman \& Bashir Yahya, (2010) "Energy efficient and QoS based routing protocol for wireless sensor networks", Journal of Parallel and Distributed Computing, Vol. 70, No. 8, pp849-857.

[8] Yanjun Yao, Qing Cao\& A. V. Vasilakos, (2015) "EDAL: An energy-efficient, delay-aware, and lifetime-balancing data collection protocol for heterogeneous wireless sensor networks", IEEE/ACM Transactions on Networking, Vol. 23, No. 3, pp810-823. 
[9] Sonam Maurya \& A. K. Daniel, (2014) "An Energy Efficient Routing Protocol under Distance, Energy and Load Parameter for Heterogeneous Wireless Sensor Networks", International Conference on Information Technology (ICIT), pp161-166.

[10] S. Li, X. Wang, S. Zhao, J. Wang \& L. Li, (2014)“'Local semidefinite programming-based node localization system for wireless sensor network applications", IEEE Systems Journal, Vol. 8, No. 3, pp879-888.

[11] F. Cadger, K. Curran, J. Santos \& S. Moffett, (2013) "A survey of geographical routing in wireless ad-hoc networks”, IEEE Communications Surveys \& Tutorials, Vol. 15, No. 2, pp621-653.

[12] J. Na, D. Soroker \& C. Kim, (2007) "Greedy geographic routing using dynamic potential field for wireless ad hoc networks", IEEE Communications Letters, Vol. 11, No. 3, pp243-245. 